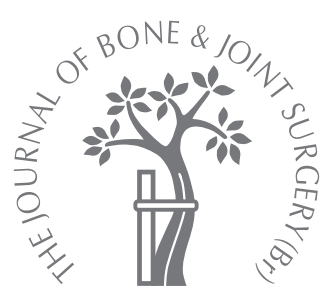

R. S. Bassi, D. Simmons, F. Ali, D. Nuttall, A. Birch, I. A. Trail, J. K. Stanley

From Wrightington Hospital, Wigan, England

\title{
Early results of the Acclaim elbow replacement
}

The Acclaim total elbow replacement is a modular system which allows implantation in both unlinked and linked modes. The results of the use of this implant in primary total elbow replacement in 36 patients, operated on between July 2000 and August 2002, are presented at a mean follow-up of 36 months (24 to 49). Only one patient did not have good relief of pain, but all had improved movement and function.

No implant showed clinical or radiological loosening, although one had a lucent area in three of seven humeral zones. The short-term results of the Acclaim total elbow replacement are encouraging. However, 11 patients $(30.5 \%)$ suffered an intra-operative fracture of the humeral condyle. This did not affect the outcome, or the requirement for further surgery, except in one case where the fracture failed to unite. This problem has hopefully been addressed by redesigning the humeral resection guide. Other complications included three cases of ulnar neuropathy $(8.3 \%)$ and one of deep infection $(2.8 \%)$.

Total elbow replacement (TER) is an established treatment for advanced rheumatoid arthritis, post-traumatic arthritis, selected distal humeral fractures and nonunions, and selected cases of ankylosis. Primary osteoarthritis of the elbow is currently an uncommon indication. ${ }^{1}$ Both linked and unlinked designs have been used, with comparable results. ${ }^{2,3}$ We describe the results of 36 patients who underwent TER using the Acclaim prosthesis (DePuy Orthopaedics Inc., Warsaw, Indiana) between July 2000 and August 2002.

\section{Patients and Methods}

We studied 46 patients (32 females and 14 males) who had undergone TER. Two patients died from unrelated causes and eight did not attend for assessment, leaving 36 available for follow-up at a mean of 36 months (24 to 49). There were 27 women and 9 men with a mean age of 64 years (34 to 80 ). The underlying disorder was rheumatoid arthritis in 32 patients, osteoarthritis in two, distal humeral nonunion in one and distal humeral fracture in one. The age of the two patients with osteoarthritis was 74 years and 78 years. The degree of arthritis was advanced; the rheumatoid patients were either Larsen's grade IV or grade $\mathrm{V}^{4}$ and the osteoarthritic patients were Kellgren's grade IV. $^{5}$

The Acclaim total elbow prosthesis has an interchangeable bearing mechanism. This allows the selection of an unlinked or a linked prosthesis (Fig. 1) before or after the operation. Both the humeral and the ulnar components are secured with cement.

All procedures were performed or directly supervised by the two senior authors (IAT and JKS). The operation was carried out under general anaesthesia with axillary nerve block, in a Charnley-Howarth ${ }^{6}$ enclosure using bodyexhaust suits, with the patient in a lateral decubitus position. Prophylactic intravenous antibiotics were administered at induction. A posterior approach was used, reflecting a tongue of the triceps aponeurosis inferiorly. ${ }^{6}$ The ulnar nerve was identified and protected, but not routinely transposed. The radial head was resected in all except one case and the posterior portion of the medial collateral ligament released. Humeral and ulnar cuts were made using a combination of a saw and burrs, with appropriate resection guides. The decision to use an unlinked or a linked prosthesis was made at this stage. An unlinked prosthesis was used in 34 patients, but because of inadequate bone stock or instability a linked prosthesis was implanted in two. After trial reduction, the humeral component was implanted, pressurising the cement against a cement restrictor after retrograde filling with a gun, a vent and finger pressure. It was not possible to use a gun to cement the ulnar compo- 


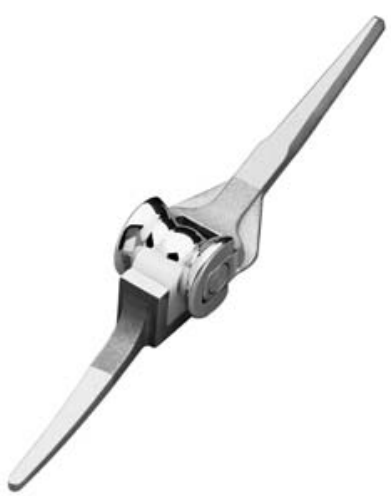

Fig. 1a

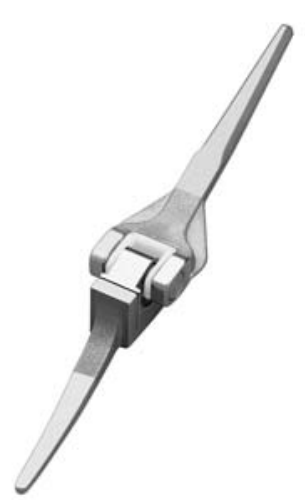

Fig. 1b
The a) unlinked and b) linked Acclaim prosthesis (C DePuy International; published with their permission).

nent, and so finger pressure was used. The skin was closed with clips over two drains and the elbow was splinted in $90^{\circ}$ of flexion. Drains were removed after 24 to 48 hours and the splint, dressings and sutures were removed at ten to 12 days post-operatively. In the majority of cases, protected movement was commenced at five days. Passive but not active extension was encouraged between two and four weeks post-operatively.

Patients were assessed clinically and radiologically preoperatively and then annually.

Follow-up evaluation. The Mayo Elbow Performance Index $^{7}$ rating for pain, range of movement, stability and function on a scale from 0 to 100 , was calculated. Function was also assessed using the American Shoulder and Elbow Surgeons (ASES) patient self-evaluation questionnaire for activities of daily living. ${ }^{8}$ The ASES score from 0 to 36 was converted to a percentage and represented on a scale from 0 to 100 . Patients were also asked to grade pain (a maximum score of 10) and satisfaction (lowest score 0 ) on a visual analogue scale from 0 to 10 . The Wrightington method $^{9}$ was used for radiological analysis of lucencies with the humeral and ulnar zones of interest distributed as illustrated in Figure 2. Radiolucencies $1 \mathrm{~mm}$ or greater were recorded.

\section{Results}

Pain relief and function as measured by the mean Mayo Elbow Performance Index and the mean ASES evaluation were improved, as were the mean ranges of movement. The results are summarised in Table $\mathrm{I}$. The mean satisfaction rating at follow-up was 9.3 (4.3 to 10$)$, and $23(64 \%)$ patients had complete relief of pain.

In $29(80.5 \%)$ of the 36 elbows, radiographs at the last follow-up showed no radiolucent lines at the cement-bone interface of either component. Radiological lucencies were

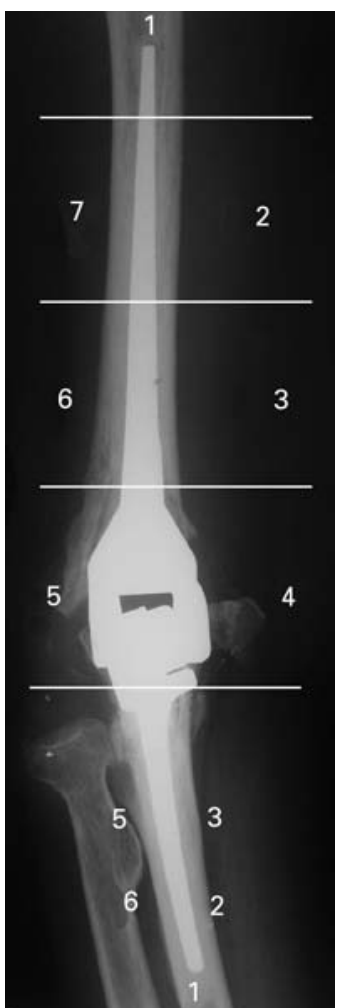

Fig. 2a

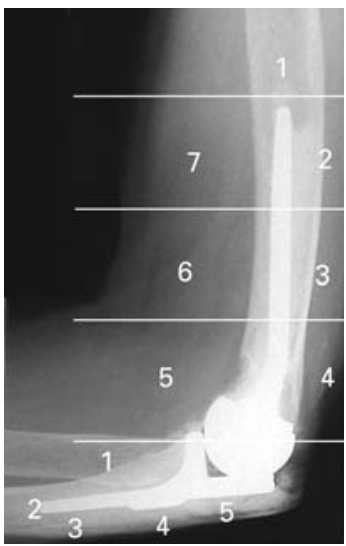

Fig. $2 b$
Wrightington distribution of humeral and ulnar radiological zones a) anteroposteriorly and b) laterally. noted in seven $(19.5 \%)$ of the elbows. In six cases only one zone was affected, and there was no progression of the lucency. In the seventh, lucencies were seen in three zones but these were not greater than $2 \mathrm{~mm}$ wide. The humeral condylar region was affected in five of the seven cases (Fig. 3). No components were found to be clinically or radiologically loose.

Complications. Intra-operative fracture occurred in 11 elbows $(30.5 \%)$. These fractures affected the medial humeral condyle in eight patients and the lateral condyle in three. Treatment involved suturing around the fracture segment back onto the implant, using No. 1 Ethibond and splintage of the elbow at $90^{\circ}$ for three weeks with no mobilisation. In one patient persistent instability resulted from a nonunion of a fracture of the lateral condyle (Fig. 4). This was revised to a linked prosthesis. One patient $(2.8 \%)$ developed a deep infection following linked TER after a supracondylar fracture of the humerus and required excision arthroplasty.

In two cases $(5.5 \%)$ subsequent peri-prosthetic fractures occurred following a fall. One minimally displaced fracture of the medial supracondylar ridge was treated with a brace for eight weeks. It united with no clinical or radiological problems. The second patient suffered a spiral fracture of the humerus just proximal to the tip of the humeral component, for which internal fixation was required. Three patients $(8.3 \%)$ suffered ulnar nerve symptoms which were transient in one but have persisted in the other two. 


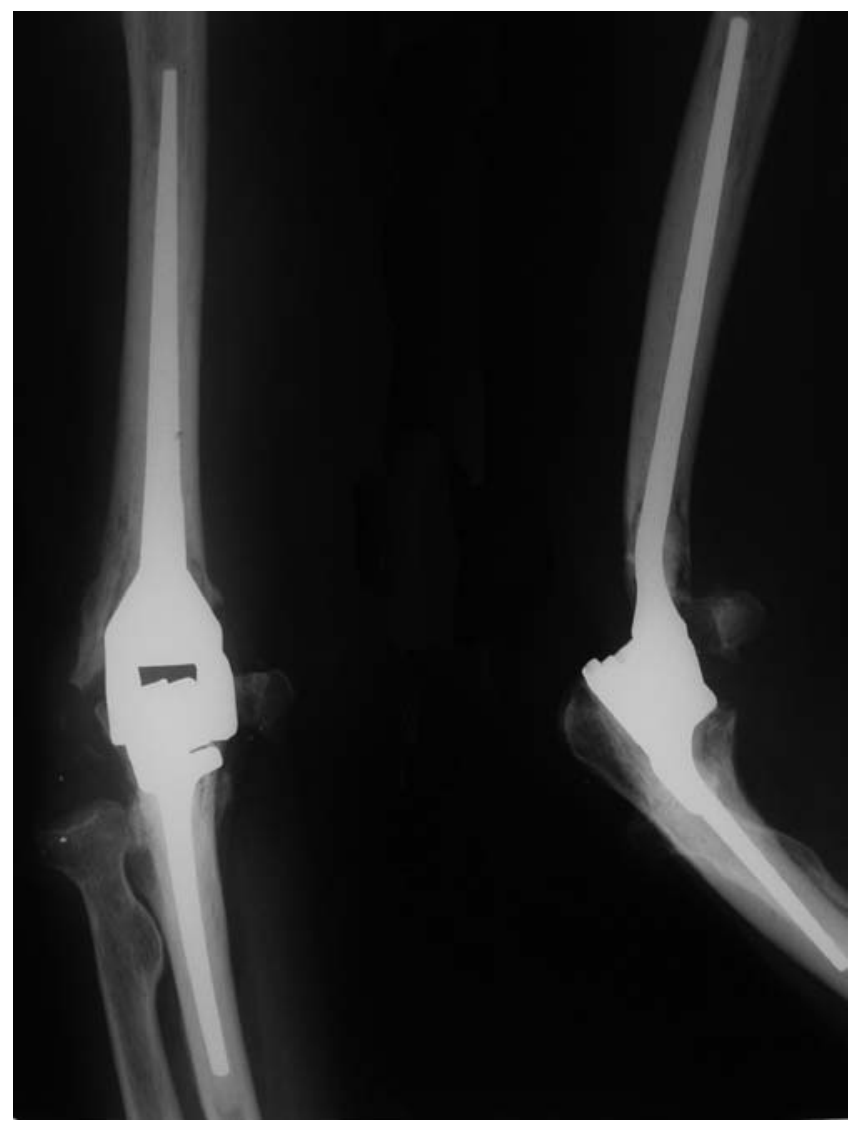

Fig. 3

Anteroposteror and lateral radiographs showing lucency at the level of the humeral condyles, and a fracture of the medial humeral condyle.

One patient who had been asymptomatic one year after operation developed pain and swelling in the elbow due to instability and required revision to a linked component at 20 months. At operation the bushings were found to be worn, which might be attributable to prolonged use of elbow crutches for problems in the lower limb. Subsequently, wound breakdown and deep infection occurred, requiring the removal of the prosthesis.

\section{Discussion}

The short-term results of the Acclaim TER show marked relief of pain and an improvement in movement and function. This is comparable to other studies. ${ }^{2,3}$ Rozing's $^{2}$ review of 33 Souter-Strathclyde elbow arthroplasties, although applying different outcome measures making direct comparison difficult, found complete pain relief in 22 of $33(67 \%)$ elbows compared with 23 of $36(64 \%)$ in our series. The mean improvement in flexion/extension was $15^{\circ}$ in his series and $23^{\circ}$ in our series. Hildebrand et $\mathrm{al}^{3}$ reviewed the functional outcomes of the Coonrad-Morrey prosthesis in 36 patients. They observed a satisfaction rating of 9.2, which is comparable to our own of 9.3. However, the mean Mayo Elbow Performance Index was 90 in

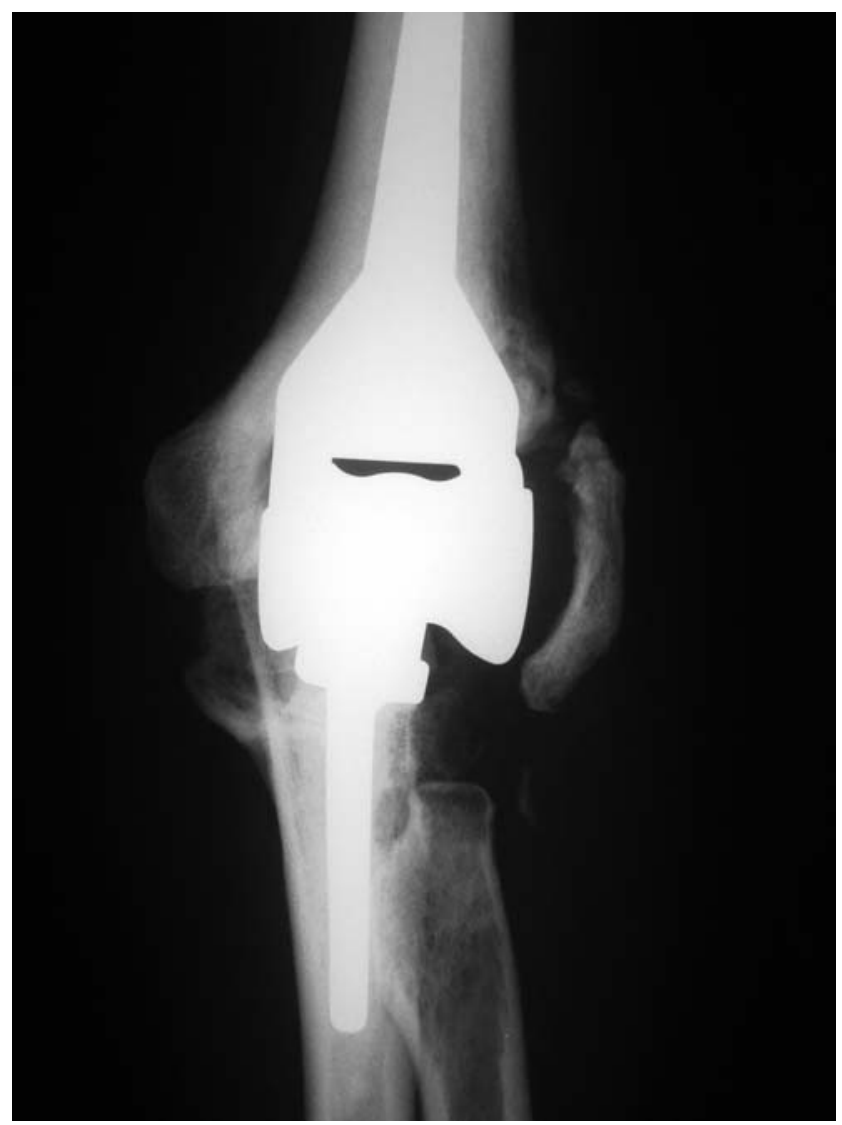

Fig. 4

Anteroposterior radiograph showing nonunion of the fracture of the lateral humeral condyle.

their subgroup who had rheumatoid arthritis, and 78 in their group who had osteoarthritis, which is superior to the overall result for our patients, who had a mean score of 76 . However, their pre-operative scores were not provided.

Our experience of one primary deep infection $(2.8 \%)$ was similar to that in Rozing's study. ${ }^{2}$ However, we encountered a higher rate of intra-operative humeral fracture, which occurred in 11 cases $(30.5 \%)$ compared with $9 \%$ in Rozing's series. ${ }^{2}$ Our incidence of three cases of ulnar neuropathy $(8.3 \%)$ was similar to that in previous reports. ${ }^{10}$

Of greater concern is the large number of intra-operative fractures of the humeral condyle. However, the Acclaim implant is a new design and we believe that the majority of these fractures were due to our inexperience in using it, as the incidence of fracture decreased later in the series. We noted that the original humeral cutting jig allowed excessive removal of the supracondylar ridges, thereby weakening the condyles. These humeral resection guides have been redesigned. Fortunately, in most cases the intra-operative fracture was managed successfully, but one patient required revision to a linked prosthesis to overcome instability resulting from nonunion of the lateral humeral condyle. A 
Table I. Summary of clinical results

\begin{tabular}{lcr}
\hline & Pre-operative & At assessment \\
\hline Mean Mayo score (range) & $36(20$ to 50$)$ & 76 (35 to 100$)$ \\
Mean ASES $^{*}$ score (range) & $34(0$ to 61$)$ & 66 (25 to 100$)$ \\
Mean pain score (range) & $8.2(0$ to 10$)$ & 2.2 (0 to 9.7$)$ \\
& & \\
Mean range of movement in ${ }^{\circ}$ (range) & & $136(110$ to 150$)$ \\
$\quad$ Flexion & $125(80$ to 140$)$ & $31(10$ to 70$)$ \\
$\quad$ Extension lag & $43(20$ to 140$)$ & $105(50$ to 130$)$ \\
$\quad$ Flexion/extension arc & $82(0$ to 110$)$ & $83(65$ to 90$)$ \\
$\quad$ Pronation & $67(30$ to 90$)$ & $76(40$ to 90$)$ \\
$\quad$ Supination & $65(20$ to 90$)$ & $159(130$ to 180$)$ \\
$\quad$ Pronation/supination arc & $132(60$ to 170$)$ & \\
\hline * ASES, American shoulder and elbow surgeons
\end{tabular}

recent study has concluded that peri-operative humeral condylar fractures cause no harmful effect. ${ }^{11}$

Two of our patients required revision for instability (5.5\%), which compares favourably with Rozing's early results with four patients $(12 \%){ }^{2}$

The early clinical results following TER using the Acclaim system are comparable to those of previous studies using other prostheses. ${ }^{2,3}$ The modular prosthesis offers the advantage of interchangeability between the unlinked and linked modes, which also makes revision easier. The frequency of intra-operative fracture, noted in these early results, has hopefully been addressed by redesigning one of the cutting guides. Medium- and long-term results are awaited.

The author or one or more of the authors have received or will receive benefits for personal or professional use from a commercial party related directly or indirectly to the subject of this article.

\section{References}

1. Kozak TK, Adams RA, Morrey BF. Total elbow arthroplasty in primary osteoarthritis of the elbow. J Arthroplasty 1998;13:837-42.
2. Rozing P. Souter-Strathclyde total elbow arthroplasty. J Bone Joint Surg $[B r]$ 2000;82-B:1129-34.

3. Hildebrand KA, Patterson SD, Regan WD, MacDermid JC, King GJ. Functional outcome of semiconstrained total elbow arthroplasty. J Bone Joint Surg [Am] 2000;82-A:1379-86.

4. Larsen A, Dale K, Eek M. Radiographic evaluation of rheumatoid arthritis and related conditions by standard reference films. Acta Radiol Diagn 1977;18:481-91.

5. Kellgren KJ, Lawrence JS. Radiological assessment of osteo-arthrosis. Ann Rheum Dis 1957;16:494-501.

6. Charnley J. A clean-air operating enclosure. Br J Surg 1964;51:202-5.

7. Crenshaw AH. Surgical techniques and approaches. In: Canale TS, ed. Campbell's operative orthopaedics. Vol. 1. Tenth ed. St Louis: Mosby, 2003:100-2.

8. Morrey BF, Adams RA. Semiconstrained arthroplasty for the treatment of rheumatoid arthritis of the elbow. J Bone Joint Surg [Am] 1992;74-A:479-90.

9. King GJ, Richards RR, Zuckerman JD, et al. A standardised method for assessment of elbow function. J Shoulder Elbow Surg 1999;8:351-4.

10. Trail IA, Nuttall D, Stanley JK. Survivorship and radiological analysis of the standard Souter-Strathclyde total elbow arthroplasty. J Bone Joint Surg [Br] 1999;81B:80-4.

11. Lyall HA, Cohen B, Clatworthy M, Constant CR. Results of the Souter Strathclyde total elbow arthroplasty in patients with rheumatoid arthritis: a preliminary report. $J$ Arthroplasty 1994;9:279-84. 